04

\title{
Электрофизическая установка для конверсии природного газа при атмосферном давлении
}

\author{
(C) В.Е. Маланичев, ${ }^{1,2}$ М.В. Малашин, ${ }^{1}$ А.В. Озерский, ${ }^{2}$ В.Ю. Хомич, ${ }^{1}$ В.М. Шмелев ${ }^{2}$ \\ ${ }^{1}$ Институт электрофизики и электроэнергетики РАН, \\ 191186 Санкт-Петербург, Россия \\ ${ }^{2}$ Институт химической фризики им. Н.Н. Семенова РАН, \\ 119991 Москва, Россия \\ e-mail: mve.191@gmail.com
}

(Поступило в Редакцию 12 декабря 2017 г. В окончательной редакции 26 апреля 2018 г.)

В электрофизической установке при обработке смеси метана и этана барьерным разрядом была экспериментально исследована конверсия углеводородов. Для разработанного плазмохимического реактора с помощью алгоритма решения „ZDPlasKin“ была проведена теоретическая оценка плазмокинетических процессов, протекающих в разряде. Экспериментальные результаты согласуются с теоретической оценкой.

DOI: 10.21883/JTF.2018.11.46625.2599

\section{Введение}

На протяжении нескольких десятилетий привлекает к себе внимание барьерный разряд с диэлектриком (БР) как средство для инициации и стимулирования химических реакций, начиная от образования озона из воздуха и заканчивая разделением различных химических соединений [1]. В неравновесной системе, которой является БР, высока эффективность и скорость проведения химических реакций [2]. При различных значениях напряженности электрического поля, или давлении среды, возбуждаются различные состояния активных частиц $[3,4]$, поэтому изменяя параметры разряда, можно направлять энергию по нужному каналу для достижения наибольшей эффективности химических реакций.

Одним из перспективных направлений является обработка метана разрядом [5,6]. В настоящее время метан в основном используется для домашнего и промышленного отопления и для выработки электроэнергии. Использование метана для химической промышленности сопровождается рядом технологических трудностей и пока ограничивается получением синтез-газа, поскольку метан является одной из наиболее стабильных молекул [7]. Синтез-газ в промышленных масштабах получают паровой конверсией метана. Также его можно получить парциальным окислением метана. Вторая технология показывает более низкий выход синтез-газа по сравнению с первой, но обладает рядом преимуществ, таких как реакция экзотермичная, процесс не требует катализатора, процесс возможно реализовать при атмосферном давлении, в процессе не используются пары воды, которые приводят к коррозии узлов установки. На основе вышеперечисленного, авторы работы считают перспективным развитие технологии с использованием реакции парциального окисления метана.

Для увеличения выхода синтез-газа при процессе парциального окисления метана предлагается предварительная обработка исходного газа (метан или природный газ) барьерным разрядом. В ряде работ [8-10] при обработке разрядом метана или метаносодержащей смеси газов наблюдался прямой синтез различных углеводородов, а также наработка химически активных частиц.

Так, в работе [8] для конверсии метана использовали импульсный искровой разряд с частотой повторения $2-20 \mathrm{kHz}$. При этом степень конверсии метана составляла $23.5 \%$, а энергозатраты - $3.8 \mathrm{eV} /$ molec. Основным продуктом плазменного пиролиза являлся ацетилен. Также искровой разряд для конверсии метана использовали авторы работы [9]. Частота повторения импульса напряжения составляла $50 \mathrm{~Hz}$ при размахе $5 \mathrm{kV}$. Зависимость конверсии метана от энерговклада была линейна и составляла от 18 до 69\% при $14-25 \mathrm{eV} / \mathrm{molec}$. В работе [10] использовался импульсный искровой разряд со схожими внешними характеристиками $(50 \mathrm{~Hz}, 5 \mathrm{kV}$ амплитуда импульсов напряжения). При этом необходимо отметить, что энергоэффективность резко уменьшилась в диапазоне, где конверсия метана составляла более чем 60\%. Таким образом, при конверсии метана 50\% энерговклад составлял $3.5 \mathrm{eV} /$ molec, а уже при $68 \%$ $12 \mathrm{eV} /$ molec.

Наиболее близкие к настоящему исследованию работы, посвященные конверсии метана в барьерном разряде. Так, в работе [9] использовали БР с двумя разными системами питания. В первом случае к электродам газоразрядной ячейки подводили униполярные прямоугольные импульсы напряжения с амплитудой до $20 \mathrm{kV}$, частотой $50 \mathrm{~Hz}$ и скважностью 2. Во втором - синусоидальное напряжение с такой же амплитудой и частотой. В первом случае конверсия метана составила 6-13\% при энергозатратах 38-57 eV/molec. Во втором - при конверсии 5-8\% энергозатраты составляли 116-175 eV/molec. В обоих случаях зависимость конверсии от энерговклада была линейна. В другой работе [10] с помощью БР, при частоте синусоидального напряжения $75 \mathrm{kHz}$, получили конверсию метана $24 \%$, энерговклад составлял $17 \mathrm{eV} / \mathrm{molec}$. 
Теоретическое моделирование процесса конверсии метана в барьерном разряде проведено в работах $[11,12]$. В первой работе конверсия метана составляла $13.8 \%$. При этом основным продуктом являлся этан. В исследовании [12] конверсия $\mathrm{CH}_{4}$ достигала $40 \%$, и в результате образовывался этан и ацетилен.

Обзор существующих теоретических и экспериментальных наработок показал, что в данный момент не существует однозначных, согласующихся результатов по процессам, происходящим при обработке метана БР. По этой причине авторы настоящей работы считают необходимым провести ряд как экспериментальных, так и теоретических исследований в этой области. Эта информация будет полезна при разработке как систем пиролиза метана в плазме для получения более сложных углеводородов, так и для систем подготовки исходной смеси для дальнейшего парциального окисления. Первичные исследования [13] были проведены для системы, в которой смешивался природный газ с воздухом. Смесь предварительно обрабатывалась плазмой и подавалась в область парциального окисления. Эти исследования показали увеличение селективности по синтез-газу. Для более эффективного использования предварительной обработки разрядом необходимо детальное понимание происходящего процесса.

В настоящей работе предпринята попытка провести сравнение экспериментально полученных результатов плазменного пиролиза природного газа с результатами 0 -мерного моделирования химической кинетики, которая инициируется и стимулируется барьерным разрядом. В качестве исходного газа использовалась смесь метана $(98.2 \%$ vol.) и этана $(1.8 \%$ vol. $)$. Такой состав смеси характерен для природного газа, подаваемого потребителям после газораспределительных станций. Таким образом, газовый состав по сравнению с предыдущими экспериментами [13] был упрощен, т. е. исключен воздух.

\section{Экспериментальная установка}

На рис. 1 схематически изображен плазмохимический реактор (ПХР) включенный в экспериментальный стенд. Входной поток $G_{g}=1.41 / \mathrm{min} 10$ поступает в семь идентичных каналов, внутри каждого из которых находится заземленный электрод 9, выполненный в виде стержня из латуни диаметром $5 \mathrm{~mm}$, стенки каналов выполнены из кварцевых трубок с внешним диаметром $10 \mathrm{~mm}$ и толщиной стенки $1.5 \mathrm{~mm} \mathrm{4}$. Каждая из трубок обвернута в алюминиевою фольгу, которая выступает в роли внешнего электрода 6 , к которому с помощью специального генератора 5 прикладывались прямоугольные высоковольтные импульсы с амплитудой $12 \mathrm{kV}$, длительностью $0.06 \mathrm{~ms}$ с частотой $f=4 \mathrm{kHz}$ [14-17]. Входной поток, проходя по каналу, попадает в область горения БР 7. Химический состав прореагировавшей смеси анализировался с помощью хроматографа марки „М3700“ 2 , который имеет три параллельных аналитических канала,

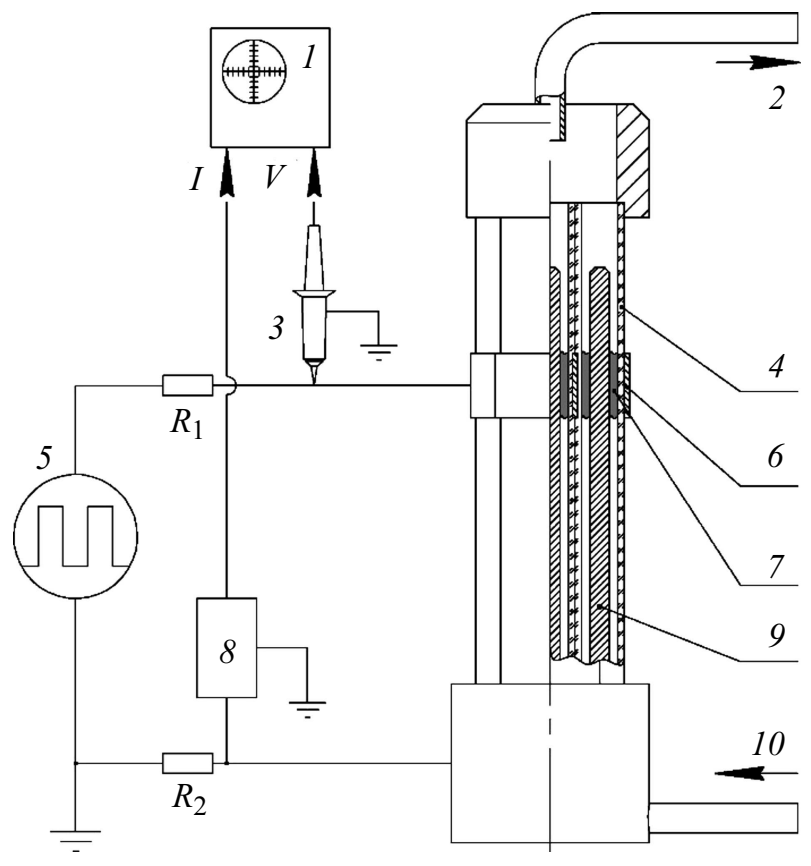

Рис. 1. Экспериментальная установка. 1 - осциллограф, $2-$ выходной поток в хроматограф, 3 - высоковольтный пробник, 4 - кварцевая трубка, 5 - генератор высоковольтных импульсов, 6 - внешний электрод, 7 - область обработки плазмой, 8 - аттенюатор, $9-$ внутренний электрод, $10-$ входной поток смеси метана и этана, $R_{1}$ - токоограничивающий резистор, $R_{2}$ - токовый шунт.

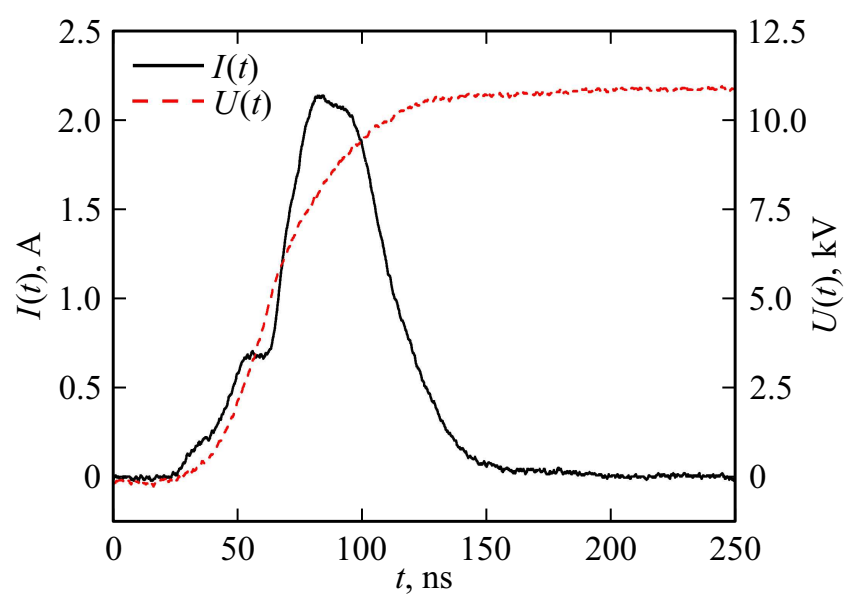

Рис. 2. Типичная осциллограмма тока $I(t)$ и зависимость от времени напряжения на разрядном промежутке $U(t)$.

что позволяет одновременно анализировать все компоненты газа. Хроматограф оснащен тремя детекторами одним пламенно-ионизационным и двумя детекторами по теплопроводности. В системе использовались набивная колонка с молекулярными ситами $\mathrm{CaA}$, колонка с Porapak Q и капиллярная колонка HP-PLOT $\mathrm{Al}_{2} \mathrm{O}_{3} / \mathrm{KCL}$, соединенная с пламенно-ионизационным детектором.

Напряжение, прикладываемое к внешнему электроду, измерялось с помощью высоковольтного пробника 
Tektronix P6015A 3. Ток, протекающий в цепи с ПХР, измерялся с помощью токового шунта $R_{2}$ и аттенюатоpa 8 .

Обработка смеси метана и этана проводилась с помощью БР при мультиканальном режиме горения разряда [18-20]. Характерные осциллограммы тока и напряжения представлены на рис. 2. Значение средней мощности, вкладываемой в плазму, составляло $25 \mathrm{~W}$. Среднее значении энергии, вкладываемой в разряд за один период, $6.2 \mathrm{~mJ}$. Среднее значение амплитуды тока разряда для каждого из семи каналов составляло $2.1 \mathrm{~A}$.

В процессе экспериментов измерялось объемное содержание метана, этилена, этана и водорода на выходе из ПХР.

\section{Теоретическая модель}

Для объяснения экспериментальных результатов проведено моделирование кинетики преобразования природного газа на основе пакета „ZDPlasKin“ [21]. Как исходные данные задавалась начальная концентрация метана $\left(N_{\mathrm{CH}_{4}}=265.1 \cdot 10^{17} \mathrm{~cm}^{-3}\right)$ и этана $\left(N_{\mathrm{C}_{2} \mathrm{H}_{6}}=4.9 \cdot 10^{17} \mathrm{~cm}^{-3}\right)$. Температура нейтральных частиц и ионов считалась постоянной и равной $300 \mathrm{~K}$.

Также в модель вводились зависимости приведенного электрического поля $E N^{-1}(t)$ и концентрации электронов $n_{e}(t)$ от времени в течение одного периода $T=1 / f=0.25 \mathrm{~ms}[3]$

$$
\begin{gathered}
E N^{-1}(t)=E_{\Sigma}(t)\left(N_{\mathrm{CH}_{4}}+N_{\mathrm{C}_{2} \mathrm{H}_{6}}\right)^{-1}, \\
n_{e}(t)=\frac{1}{q_{e} \mu_{e} S_{\mathrm{md}} E_{\Sigma}(t)} .
\end{gathered}
$$

Здесь $E_{\Sigma}(t)-$ суммарная напряженность электрического поля, $q_{e}-$ заряд электрона, $\mu_{e}=$ $=280 \mathrm{~cm}^{2}(\mathrm{~V} \times \mathrm{s})^{-1}-$ среднее значение подвижности электронов в метане $[22,23], I(t)-$ ток, протекающий через разрядный промежуток, $S_{\mathrm{md}}-$ суммарная

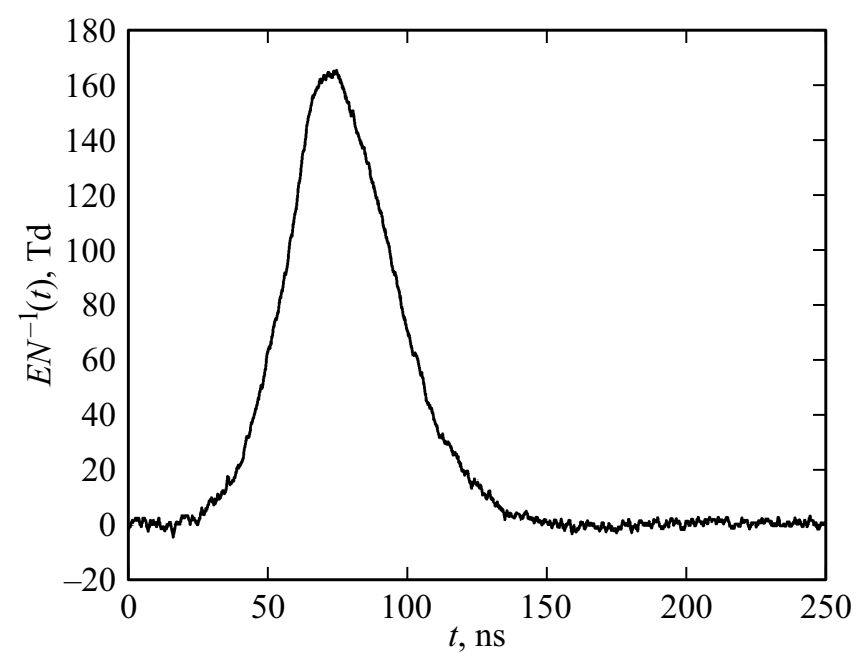

Рис. 3. Зависимость приведенной напряженности электрического поля от времени.

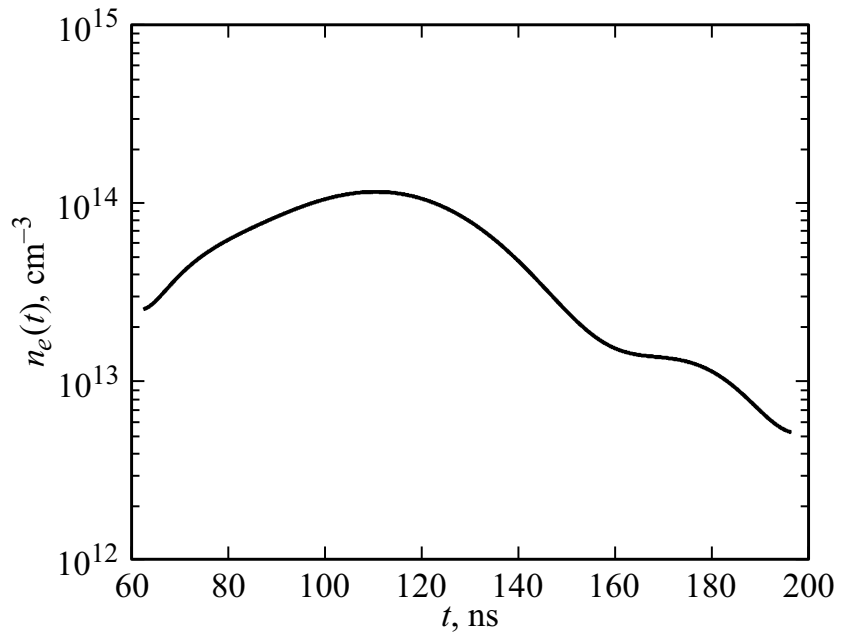

Рис. 4. Зависимость концентрации электронов от времени.

площадь поперечных сечений микроразрядов, происходящих за время переднего/заднего фронта импульса напряжения. Количество микроразрядов оценивалось исходя из данных работы [24] о заряде, переносимом одним микроразрядом $Q_{\mathrm{md}}=0.5 \mathrm{nC}$. Суммарный заряд $Q_{\Sigma}=116 \mathrm{nC}$, полученный из осциллограммы тока (рис. 2), делился на $Q_{\mathrm{md}}$. Диаметр поперечного сечения одного микроразряда $d_{c}=0.3 \mathrm{~mm}$ был взят из работы [20]. Таким образом,

$$
S_{\mathrm{md}}=\frac{Q_{\Sigma}}{Q_{\mathrm{md}}} \frac{\pi d_{c}^{2}}{4} .
$$

Суммарная напряженность электрического поля рассчитывалась исходя из типичной осциллограммы напряжения (рис. 2), на разрядном промежутке, по формуле

$$
E_{\Sigma}=\frac{U(t)}{l}-E_{\mathrm{com}}
$$

где $U(t)$ - напряжение на разрядном промежутке, $l=1 \mathrm{~mm}-$ межэлектродный зазор, $E_{\mathrm{com}}-$ напряженность электрического поля, созданная зарядом, осевшим на диэлектрик. Напряженность электрического поля в зазоре рассчитывалась по формуле для промежутка с плоско-параллельной геометрией. Такое приближение уместно для коаксиальной геометрии с отношением кривизны электродов, близким к единице. Напряженность электрического поля $E_{\text {com }}$ рассчитывалась по следующей формуле:

$$
E_{\mathrm{com}}=\frac{\int_{t_{0}}^{t} l(t)}{\varepsilon_{0} \varepsilon_{\mathrm{CH}_{4}} S},
$$

где $t_{0}-$ момент начала зарядно-разрядных процессов, $\varepsilon_{0}$ - электрическая постоянная, $\varepsilon_{\mathrm{CH}_{4}}$ - диэлектрическая проницаемость метана, $S=220 \mathrm{~mm}^{2}-$ площадь внутренней поверхности диэлектрика, на которой накапливается заряд. 
Таблица 1. Процессы, включенные в модель

\begin{tabular}{|c|c|c|c|}
\hline \multicolumn{4}{|c|}{ Группа № 1} \\
\hline Процесс & Ссылка & Процесс & Ссылка \\
\hline \multicolumn{2}{|c|}{ Упругие соударения } & \multicolumn{2}{|c|}{ Диссоциация } \\
\hline $\begin{array}{l}\mathrm{CH}_{4}+\mathrm{e} \rightarrow \mathrm{CH}_{4}+\mathrm{e} \\
\mathrm{C}_{2} \mathrm{H}_{6}+\mathrm{e} \rightarrow \mathrm{C}_{2} \mathrm{H}_{6}+\mathrm{e}\end{array}$ & $\begin{array}{l}{[23]} \\
{[28]}\end{array}$ & $\begin{array}{l}\mathrm{CH}_{4}+\mathrm{e} \rightarrow \mathrm{CH}_{3}+\mathrm{H}+\mathrm{e} \\
\mathrm{CH}_{4}+\mathrm{e} \rightarrow \mathrm{CH}_{2}+\mathrm{H}_{2}+\mathrm{e}\end{array}$ & $\begin{array}{l}{[31]} \\
{[31]}\end{array}$ \\
\hline \multicolumn{2}{|c|}{ Ионизация } & $\mathrm{CH}_{4}+\mathrm{e} \rightarrow \mathrm{CH}+\mathrm{H}+\mathrm{H}_{2}+\mathrm{e}$ & {$[31]$} \\
\hline $\begin{array}{l}\mathrm{CH}_{4}+\mathrm{e} \rightarrow \mathrm{CH}_{4}^{+}+2 \mathrm{e} \\
\mathrm{CH}_{4}+\mathrm{e} \rightarrow \mathrm{CH}_{3}^{+}+2 \mathrm{e}+\mathrm{H} \\
\mathrm{C}_{2} \mathrm{H}_{6}+\mathrm{e} \rightarrow \mathrm{C}_{2} \mathrm{H}_{6}^{+}+2 \mathrm{e} \\
\mathrm{C}_{2} \mathrm{H}_{4}+\mathrm{e} \rightarrow \mathrm{C}_{2} \mathrm{H}_{4}^{+}+2 \mathrm{e} \\
\mathrm{C}_{2} \mathrm{H}_{2}+\mathrm{e} \rightarrow \mathrm{C}_{2} \mathrm{H}_{2}^{+}+2 \mathrm{e} \\
\mathrm{C}_{3} \mathrm{H}_{8}+\mathrm{e} \rightarrow \mathrm{C}_{3} \mathrm{H}_{8}^{+}+2 \mathrm{e} \\
\mathrm{C}_{3} \mathrm{H}_{6}+\mathrm{e} \rightarrow \mathrm{C}_{3} \mathrm{H}_{6}^{+}+2 \mathrm{e} \\
\mathrm{H}_{2}+\mathrm{e} \rightarrow \mathrm{H}_{2}^{+}+2 \mathrm{e}\end{array}$ & $\begin{array}{l}{[23]} \\
{[23]} \\
{[23]} \\
{[23]} \\
{[23]} \\
{[29]} \\
{[30]} \\
{[31]}\end{array}$ & $\begin{array}{l}\mathrm{C}_{2} \mathrm{H}_{6}+\mathrm{e} \rightarrow \mathrm{C}_{2} \mathrm{H}_{5}+\mathrm{H}+\mathrm{e} \\
\mathrm{C}_{2} \mathrm{H}_{6}+\mathrm{e} \rightarrow \mathrm{C}_{2} \mathrm{H}_{4}+\mathrm{H}_{2}+\mathrm{e} \\
\mathrm{C}_{2} \mathrm{H}_{4}+\mathrm{e} \rightarrow \mathrm{C}_{2} \mathrm{H}_{3}+\mathrm{H}+\mathrm{e} \\
\mathrm{C}_{2} \mathrm{H}_{2}+\mathrm{e} \rightarrow \mathrm{CH}+\mathrm{CH}+\mathrm{e} \\
\mathrm{C}_{2} \mathrm{H}_{2}+\mathrm{e} \rightarrow \mathrm{C}_{2} \mathrm{H}+\mathrm{H}+\mathrm{e} \\
\mathrm{C}_{2} \mathrm{H}_{2}+\mathrm{e} \rightarrow \mathrm{CH}_{2}+\mathrm{C}+\mathrm{e} \\
\mathrm{C}_{3} \mathrm{H}_{8}+\mathrm{e} \rightarrow \mathrm{C}_{2} \mathrm{H}_{4}+\mathrm{CH}_{4}+\mathrm{e} \\
\mathrm{C}_{3} \mathrm{H}_{8}+\mathrm{e} \rightarrow \mathrm{C}_{3} \mathrm{H}_{6}+\mathrm{H}_{2}+\mathrm{e} \\
\mathrm{H}_{2}+\mathrm{e} \rightarrow \mathrm{H}+\mathrm{H}+\mathrm{e}\end{array}$ & $\begin{array}{l}{[32]} \\
{[32]} \\
{[32]} \\
{[32]} \\
{[32]} \\
{[32]} \\
{[32]} \\
{[32]} \\
{[33]}\end{array}$ \\
\hline
\end{tabular}

Группа № 2

\begin{tabular}{|c|c|c|}
\hline Процесс & Коэф. скорости реакции & Ссылка \\
\hline $\begin{array}{l}\mathrm{CH}_{4}+\mathrm{CH} \rightarrow \mathrm{C}_{2} \mathrm{H}_{4}+\mathrm{H} \\
\mathrm{CH}_{4}+\mathrm{CH}_{2} \rightarrow \mathrm{CH}_{3}+\mathrm{CH}_{3} \\
\mathrm{CH}_{4}+\mathrm{C}_{2} \mathrm{H} \rightarrow \mathrm{C}_{2} \mathrm{H}_{2}+\mathrm{CH}_{3} \\
\mathrm{CH}_{4}+\mathrm{C}_{2} \mathrm{H}_{3} \rightarrow \mathrm{C}_{2} \mathrm{H}_{4}+\mathrm{CH}_{3} \\
\mathrm{CH}_{4}+\mathrm{C}_{2} \mathrm{H}_{5} \rightarrow \mathrm{C}_{2} \mathrm{H}_{6}+\mathrm{CH}_{3} \\
\mathrm{CH}_{4}+\mathrm{H} \rightarrow \mathrm{H}_{2}+\mathrm{CH}_{3} \\
\mathrm{CH}_{4}+\mathrm{C}_{2} \mathrm{H}_{2}+\mathrm{H} \rightarrow \mathrm{C}_{2} \mathrm{H}_{3}+\mathrm{CH}_{4} \\
\mathrm{CH}_{4}+\mathrm{C}_{2} \mathrm{H}_{4}+\mathrm{H} \rightarrow \mathrm{C}_{2} \mathrm{H}_{5}+\mathrm{CH}_{4} \\
\mathrm{CH}_{4}+\mathrm{C}_{2} \mathrm{H}_{5}+\mathrm{H} \rightarrow \mathrm{C}_{2} \mathrm{H}_{6}+\mathrm{CH}_{4} \\
\mathrm{CH}_{3}+\mathrm{CH}_{3} \rightarrow \mathrm{C}_{2} \mathrm{H}_{5}+\mathrm{H} \\
\mathrm{CH}_{3}+\mathrm{C}_{2} \mathrm{H}_{2} \rightarrow \mathrm{C}_{2} \mathrm{H}+\mathrm{CH}_{4} \\
\mathrm{CH}_{3}+\mathrm{C}_{2} \mathrm{H}_{4} \rightarrow \mathrm{C}_{2} \mathrm{H}_{3}+\mathrm{CH}_{4} \\
\mathrm{CH}_{3}+\mathrm{CH}_{3}+\mathrm{CH}_{4} \rightarrow \mathrm{C}_{2} \mathrm{H}_{6}+\mathrm{CH}_{4} \\
\mathrm{CH}_{3}+\mathrm{C}_{2} \mathrm{H}_{5}+\mathrm{CH}_{4} \rightarrow \mathrm{C}_{3} \mathrm{H}_{8}+\mathrm{CH}_{4} \\
\mathrm{CH}_{2}+\mathrm{H}_{2} \rightarrow \mathrm{H}+\mathrm{CH}_{3} \\
\mathrm{C}_{2} \mathrm{H}+\mathrm{C}_{2} \mathrm{H}_{2} \rightarrow \mathrm{C}_{4} \mathrm{H}_{2}+\mathrm{H} \\
\mathrm{C}_{2} \mathrm{H}_{2}+\mathrm{H}_{2} \rightarrow \mathrm{C}_{2} \mathrm{H}+\mathrm{H}_{2} \\
\mathrm{C}_{2} \mathrm{H}_{3}+\mathrm{C}_{2} \mathrm{H}_{3} \rightarrow \mathrm{C}_{2} \mathrm{H}_{4}+\mathrm{C}_{2} \mathrm{H}_{2} \\
\mathrm{C}_{2} \mathrm{H}_{3}+\mathrm{H}_{2} \rightarrow \mathrm{C}_{2} \mathrm{H}_{4}+\mathrm{H} \\
\mathrm{C}_{2} \mathrm{H}_{5}+\mathrm{C}_{2} \mathrm{H}_{5} \rightarrow \mathrm{C}_{2} \mathrm{H}_{6}+\mathrm{C}_{2} \mathrm{H}_{4} \\
\mathrm{C}_{2} \mathrm{H}_{5}+\mathrm{H}_{2} \rightarrow \mathrm{C}_{2} \mathrm{H}_{6}+\mathrm{H}\end{array}$ & $\begin{array}{l}9.74 \times 10^{-11} \mathrm{~cm}^{3} \mathrm{~s}^{-1} \\
3.01 \times 10^{-19} \mathrm{~cm}^{3} \mathrm{~s}^{-1} \\
1.31 \times 10^{-12} \mathrm{~cm}^{3} \mathrm{~s}^{-1} \\
2.28 \times 10^{-18} \mathrm{~cm}^{3} \mathrm{~s}^{-1} \\
1.83 \times 10^{-24} \mathrm{~cm}^{3} \mathrm{~s}^{-1} \\
8.43 \times 10^{-19} \mathrm{~cm}^{3} \mathrm{~s}^{-1} \\
2.81 \times 10^{-31} \mathrm{~cm}^{6} \mathrm{~s}^{-1} \\
3.66 \times 10^{-30} \mathrm{~cm}^{6} \mathrm{~s}^{-1} \\
9.20 \times 10^{-30} \mathrm{~cm}^{6} \mathrm{~s}^{-1} \\
2.71 \times 10^{-19} \mathrm{~cm}^{3} \mathrm{~s}^{-1} \\
7.65 \times 10^{-26} \mathrm{~cm}^{3} \mathrm{~s}^{-1} \\
1.94 \times 10^{-21} \mathrm{~cm}^{3} \mathrm{~s}^{-1} \\
1.56 \times 10^{-26} \mathrm{~cm}^{6} \mathrm{~s}^{-1} \\
1.00 \times 10^{-28} \mathrm{~cm}^{6} \mathrm{~s}^{-1} \\
5.00 \times 10^{-15} \mathrm{~cm}^{3} \mathrm{~s}^{-1} \\
1.50 \times 10^{-10} \mathrm{~cm}^{3} \mathrm{~s}^{-1} \\
6.12 \times 10^{-27} \mathrm{~cm}^{3} \mathrm{~s}^{-1} \\
1.60 \times 10^{-12} \mathrm{~cm}^{3} \mathrm{~s}^{-1} \\
9.78 \times 10^{-20} \mathrm{~cm}^{3} \mathrm{~s}^{-1} \\
2.41 \times 10^{-12} \mathrm{~cm}^{3} \mathrm{~s}^{-1} \\
2.97 \times 10^{-21} \mathrm{~cm}^{3} \mathrm{~s}^{-1}\end{array}$ & $\begin{array}{l}{[12]} \\
{[12]} \\
{[12]} \\
{[12]} \\
{[12]} \\
{[12]} \\
{[12]} \\
{[12]} \\
{[12]} \\
{[12]} \\
{[12]} \\
{[12]} \\
{[12]} \\
{[12]} \\
{[12]} \\
{[12]} \\
{[12]} \\
{[12]} \\
{[12]} \\
{[12]} \\
{[12]}\end{array}$ \\
\hline
\end{tabular}

\begin{tabular}{l}
\hline $\mathrm{CH}_{5}^{+}+\mathrm{C}_{2} \mathrm{H}_{4} \rightarrow \mathrm{C}_{2} \mathrm{H}_{5}^{+}+\mathrm{CH}_{4}$ \\
$\mathrm{CH}_{5}^{+}+\mathrm{C}_{2} \mathrm{H}_{2} \rightarrow \mathrm{C}_{2} \mathrm{H}_{5}^{+}+\mathrm{CH}_{4}$ \\
$\mathrm{CH}_{4}^{+}+\mathrm{CH}_{4} \rightarrow \mathrm{CH}_{5}^{+}+\mathrm{CH}_{3}$ \\
$\mathrm{CH}_{3}^{+}+\mathrm{CH}_{4} \rightarrow \mathrm{C}_{2} \mathrm{H}_{5}^{+}+\mathrm{H}_{2}$ \\
$\mathrm{C}_{2} \mathrm{H}_{5}^{+}+\mathrm{H} \rightarrow \mathrm{C}_{2} \mathrm{H}_{4}^{+}+\mathrm{H}_{2}$ \\
$\mathrm{C}_{2} \mathrm{H}_{4}^{+}+\mathrm{C}_{2} \mathrm{H}_{3} \rightarrow \mathrm{C}_{2} \mathrm{H}_{5}^{+}+\mathrm{C}_{2} \mathrm{H}_{2}$ \\
$\mathrm{C}_{2} \mathrm{H}_{4}^{+}+\mathrm{C}_{2} \mathrm{H}_{3} \rightarrow \mathrm{C}_{2} \mathrm{H}_{3}^{+}+\mathrm{C}_{2} \mathrm{H}_{4}$ \\
$\mathrm{C}_{2} \mathrm{H}_{4}^{+}+\mathrm{H} \rightarrow \mathrm{C}_{2} \mathrm{H}_{3}^{+}+\mathrm{H}_{2}$
\end{tabular}

Результат расчета зависимостей $E N^{-1}(t)$ и $n_{e}(t)$, соответствующих времени развития разряда при нарастании напряжения, представлены на рис. 3 и 4. При расчетах
Группа № 3

$1.50 \times 10^{-9} \mathrm{~cm}^{3} \mathrm{~s}^{-1}$
$1.60 \times 10^{-9} \mathrm{~cm}^{3} \mathrm{~s}^{-1}$
$1.50 \times 10^{-9} \mathrm{~cm}^{3} \mathrm{~s}^{-1}$
$1.20 \times 10^{-9} \mathrm{~cm}^{3} \mathrm{~s}^{-1}$
$1.00 \times 10^{-11} \mathrm{~cm}^{3} \mathrm{~s}^{-1}$
$5.00 \times 10^{-10} \mathrm{~cm}^{3} \mathrm{~s}^{-1}$
$5.00 \times 10^{-10} \mathrm{~cm}^{3} \mathrm{~s}^{-1}$
$3.00 \times 10^{-10} \mathrm{~cm}^{3} \mathrm{~s}^{-1}$

используется 0-мерное приближение, т.е. рассчитывались параметры $E N^{-1}(t)$ и $n_{e}(t)$ для малого объема, который находился в начале (на фронте ионизации) 
Таблица 2. Частицы, включенные в модель

\begin{tabular}{c|c|c}
\hline Молекулы & Ионы & Радикалы \\
\hline $\mathrm{CH}_{4}, \mathrm{C}_{2} \mathrm{H}_{2}, \mathrm{C}_{2} \mathrm{H}_{4}, \mathrm{C}_{2} \mathrm{H}_{6}$, & $\mathrm{CH}_{3}^{+}, \mathrm{CH}_{4}^{+}, \mathrm{CH}_{4}^{+}, \mathrm{C}_{2} \mathrm{H}_{2}^{+}$, & $\mathrm{C}_{2} \mathrm{CH}_{2} \mathrm{CH}_{2}, \mathrm{CH}_{3}, \mathrm{C}_{2} \mathrm{H}$, \\
$\mathrm{C}_{3} \mathrm{H}_{6}, \mathrm{C}_{3} \mathrm{H}_{8}, \mathrm{C}_{4} \mathrm{H}_{2}, \mathrm{H}_{2}$ & $\mathrm{C}_{2} \mathrm{H}_{3}^{+}, \mathrm{C}_{2} \mathrm{H}_{4}^{+}, \mathrm{C}_{2} \mathrm{H}_{5}^{+}, \mathrm{C}_{2} \mathrm{H}_{6}^{+}$, & $\mathrm{C}_{2} \mathrm{H}_{3}, \mathrm{C}_{2} \mathrm{H}_{5}, \mathrm{H}$
\end{tabular}

Таблица 3. Сравнение результатов анализа химического состава хроматографом и результатов моделирования

\begin{tabular}{l|c|r|r}
\hline Компонент & Исходный состав & Эксперимент & Моделирование \\
\hline$N_{\mathrm{CH}_{4}}, 10^{17}, \mathrm{~cm}^{-3}$ & $265.2 \pm 0.3$ & $260.5 \pm 1.4$ & 262.4 \\
$N_{\mathrm{C}_{2} \mathrm{H}_{4}}, 10^{17}, \mathrm{~cm}^{-3}$ & 0.0 & $2.4 \pm 0.4$ & 2.6 \\
$N_{\mathrm{C}_{2} \mathrm{H}_{6}}, 10^{17}, \mathrm{~cm}^{-3}$ & $4.8 \pm 0.4$ & $5.9 \pm 0.5$ & 6.3 \\
$N_{\mathrm{H}_{2}}, 10^{17}, \mathrm{~cm}^{-3}$ & 0.0 & $1.2 \pm 0.4$ & 1.5
\end{tabular}

стримера в момент его „прорастания“. Формирование каждого такого канала происходит по классическому стримерному механизму, описанному Б. Лёбом [25], с поправкой на наличие диэлектрика на поверхности металлического электрода. Такой механизм развития разряда подтверждается рядом экспериментов с оптической регистрацией процесса развития разряда [26,27]. В этом объеме наибольшая концентрация электронов, а также на заряженные частицы действует электрическое поле большей величины (подобно системе „игла-плоскость“). В работе [25] утверждается, что стример развивается при напряженности этого поля, равной значению напряженности внешнего приложенного электрического поля.

Исходя из расхода газа $G_{\mathrm{g}}$ и геометрии разрядного промежутка рассчитывалось время нахождения обрабатываемого газа в разрядном промежутке $t_{\text {res }}=57 \mathrm{~ms}$. Таким образом, количество импульсов $N_{\mathrm{imp}}=t_{\text {res }} / T=228$.

В модели расчет был рассмотрен еще в течение $300 \mathrm{~s}$ после подачи последнего импульса, что соответствовало времени диагностики, во время которого шли реакции рекомбинации активных радикалов. При этом значения $n_{e}(t)$ и $E N^{-1}(t)$ были близки к нулю.

При моделировании учитывался 51 процесс (табл. 1) в котором участвовало 27 частиц, не считая электронов (табл. 2). В табл. 1 все процессы разделены на три группы. Группа № 1 включает в себя столкновения электронов с различными молекулами и радикалами. Группа № 2 - реакции при столкновении двух нейтральных частиц. В группе № 3 указаны процессы взаимодействия нейтральных частиц и ионов. Также в таблице для каждой реакции указан источник откуда была взята для расчета зависимость поперечного сечения от энергии электронов или скоростной коэффициент.

\section{Результаты и обсуждение}

В результате моделирования были получены зависимости концентрации рассматриваемых частиц от вре-

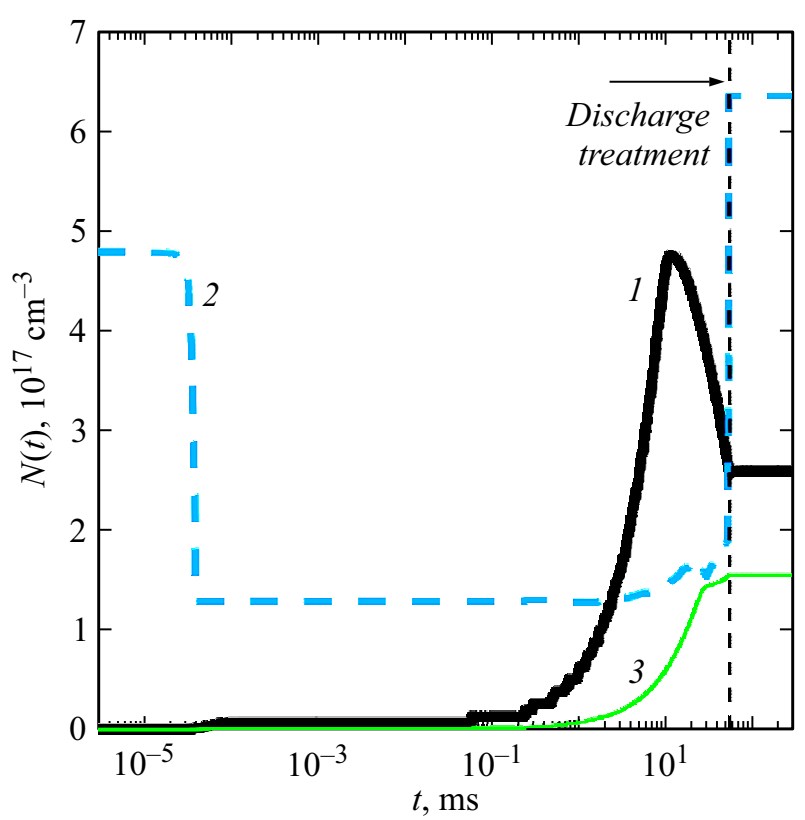

Рис. 5. Результаты моделирования зависимости концентрации молекул от времени $1-\mathrm{C}_{2} \mathrm{H}_{4} ; 2-\mathrm{C}_{2} \mathrm{H}_{6} ; 3-\mathrm{H}_{2}$.

мени. На рис. 5 приведены зависимости $N(t)$, полученные в результате моделирования для молекул, которые сравнивались с экспериментальными данными $\left(\mathrm{C}_{2} \mathrm{H}_{4}, \mathrm{C}_{2} \mathrm{H}_{6}, \mathrm{H}_{2}\right)$. Изменение хода графика зависимости $N_{\mathrm{C}_{2} \mathrm{H}_{4}}(t)$ объясняется увеличением скорости реакции потребления этилена из-за увеличения концентрации атомарного водорода, который участвует в реакции $\mathrm{C}_{2} \mathrm{H}_{4}+\mathrm{H}+\mathrm{CH}_{4} \rightarrow \mathrm{C}_{2} \mathrm{H}_{5}+\mathrm{CH}_{4}$. Резкое увеличение концентрации этана связано с рекомбинацией остаточных радикалов $\mathrm{CH}_{3}$ и $\mathrm{C}_{2} \mathrm{H}_{5}$ после окончания всех разрядных процессов.

В табл. 3 представлены обработанные (\% vol. переведены в $\mathrm{cm}^{-3}$ ) результаты анализа химического состава газа с помощью хроматографа и результаты моделиро- 


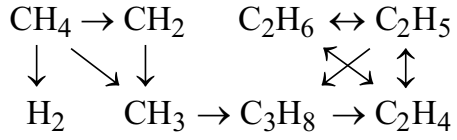

Pис. 6. Схема основных превращений, инициированных барьерным разрядом.

вания. Для результатов моделирования приведено значение концентраций в заключительный момент времени.

Экспериментальные результаты показывают, что в процессе плазменного пиролиза природного газа, основной продукт - этилен (его селективность составила $60.1 \%$ ). Также в составе на выходе из плазмохимического реактора обнаружены этан и водород (их селективности 30 и $8.8 \%$ соответственно). Необходимо отметить, что в процессе экспериментов количество этана в смеси не всегда изменялось в сторону увеличения, т. е. в части экспериментов количество этана не изменялось либо незначительно уменьшалось. В результатах представлено среднее значение ряда экспериментов.

Значение селективности по этилену, полученное в настоящей работе, превышает то же значение в работах $[9,10]$. При этом эксперименты проводились в диапазоне более низкого энерговклада $(0.5 \mathrm{eV} / \mathrm{molec})$, чем в работе [9] (38-175 eV/molec).

В табл. 3 значение концентраций в результате моделирования отличается от результатов эксперимента. Это объясняется тем, что в модели рассчитывались только газофазные процессы, в то время как присутствовали реакции, приводящие к образованию осаждений на поверхности внутренних электродов и диэлектриков. В работе [34] исследовался состав подобной плёнки, полученной в результате воздействия БР на метан. В результате исследования выяснилось, что пленка имеет сложный состав, подобный промышленному минеральному маслу.

Анализируя полученные результаты, были предложены наиболее вероятные пути химических превращений, по которым происходили процессы в системе. Они представлены схематически на рис. 6. В схему введены углеводороды $\mathrm{C}_{3}$, которые в процессе эксперимента не измерялись, но использовались при моделировании. Необходимо отметить, что на схеме опущены некоторые обратные связи, например, при образовании этилена из пропана образуется метан. Полученные результаты хорошо согласуются с прежними наработками по этой тематике $[11,12]$.

\section{Заключение}

В процессе экспериментов обрабатывалась смесь метана и этана барьерным разрядом. Основным продуктом являлся этилен (селективность 60.1\%). С помощью алгоритма решения „ZDPlasKin“ была проведена теоретическая оценка плазмокинетических процессов, протекающих в разряде.

Из табл. 3 видно, что результаты эксперимента и моделирования согласуются. Таким образом, можно сделать вывод, что 0-мерная плазмокинетическая модель приемлема для оценки наиболее вероятных путей протекания реакций, стимулированных барьерным разрядом в плазмохимическом реакторе. Зная необходимый химический состав выходного потока из реактора и состав исходной смеси, с помощью этой модели возможно подобрать зависимости $E N^{-1}(t), n_{e}(t) t_{\text {res. }}$ Исходя из этих параметров рассчитываются параметры электрофизической установки, такие как: $U(t), I(t), f$, геометрия разрядного промежутка и расход исходного газа.

Работа выполнена при поддержке Российского фонда фундаментальных исследований (грант № 16-08-01037-A).

\section{Список литературы}

[1] Самойлович В.Г., Гибалов В.И., Козлов К.В. Физическая химия барьерного разряда. М.: Изд-во МГУ, 1989. 176 с.

[2] Русанов В.Д., Фридман А.А., Шолин Г.В. // УФН. 1981. T. 134. Вып. 6. C. 185-235. [Rusanov V.D., Fridman A.A., Sholin G.V. // Sov. Phys. Usp. 1981. Vol. 24. N 6. P. 447-474.] DOI: $10.3367 /$ UFNr.0134.198106a.0185

[3] Райзер Ю.П. Физика газового разряда. М.: Наука, 1987. $592 \mathrm{c}$.

[4] Чукаловский А.А., Рахимова Т.В., Клоповский К.С., Попов Н.А., Манкелевич Ю.А., Прочина О.В. // Физика плазмы. 2014. Т. 40 Вып. 1. С. 63-76. DOI: $10.7868 / \mathrm{S} 0367292114010041$

[5] Tan X., Thursfield A., Metcalfe I.S., Li K. // Asia-Pacific J. of Chem. Engineer. 2009. Vol. 4. N 3. P. 251.

[6] Munera J.F., Coronel L., Faroldi B., Carrara C., Lombardo E.A., Cornaglia L.M. // Asia-Pacific J. Chem. Engineer. 2010. Vol. 5. N 1. P. 35.

[7] Lunsford J.H. // Catal. Today. 2000. Vol. 63. P. 165.

[8] Yao S.L., Suzuki E., Meng N., Nakayama A. // Plasma Chemistry and Plasma Processing. 2002. Vol. 22. N 2. P. 225.

[9] Li X., Zhu A., Wanga K., Xua Y., Song Z. // Catal. Today. 2004. Vol. 98. P. 617.

[10] Kado S., Sekine Y., Muto N., Nozaki T., Okazaki K. // Proc. 16th Intern. Symp. on Plasma Chem. Taormina. 2003. P. 406.

[11] Indarto A., Coowanitwong N., Choi J., Lee H., Song H. // Fuel Processing Technology. 2008. Vol. 89. N 2. P. 214.

[12] Bie C.D., Verheyde B., Martens T., Dijk J., Paulussen S., Bogaerts A. // Plasma Process. Polym. 2011. Vol. 8. P. 1033.

[13] Маланичев В.Е., Малашин М.В., Мошкунов С.И., Небогаткин С.В., Хомич В.Ю., Шмелев В.М. // Письма в ЖТФ. 2017. Т. 43. Вып. 10. С. 12-17. [Malanichev V.E., Malashin M.V., Moshkunov S.I., Nebogatkin S.V., Khomich V.Yu., Shmelev V.M. // Tech. Phys. Lett. 2017. Vol. 43. N 5. P. 460-462.] DOI: 10.21883 /PJTF.2017.10.44615.16613

[14] Иванов Е.В., Мошкунов С.И., Хомич В.Ю. // Прикладная физика. 2006. Вып. 2. С. 122.

[15] Иванов Е.В., Мошкунов С.И., Хомич В.Ю. // Приборы и техника эксперимента. 2006. Вып. 1. С. 88. 
[16] Малашин М.В., Мошкунов С.И., Хомич В.Ю., Шериунова E.A. // Приборы и техника эксперимента. 2016. Вып. 2. C. 71.

[17] Малашин М.В., Мошкунов С.И., Хомич В.Ю., Шершунова Е.А. // Письма в ЖТФ. 2015. Т. 41. Вып. 9. С. 54.

[18] Малашин М.В., Мошкунов С.И., Хомич В.Ю., Шериунова Е.А., Ямщиков В.А. // Письма в ЖТФ. 2013. Т. 39. Вып. 5. С. 48.

[19] Khomich V.Yu., Malashin M.V., Moshkunov S.I., Shershunova E.A., Yamschikov V.A. // IEEE Trans. on Plasma Sci. 2014. Vol. 42. N 10. P. 3314.

[20] Khomich V.Yu., Malanichev V.E., Malashin M.V., Moshkunov S.I. // IEEE Trans. on Plasma Sci. Vol. 44. N 8. P. 1349.

[21] Pancheshnyi S., Eismann B., Hagelaar G.J.M., Pitchford L.C. Computer code ZDPlasKin, http://www.zdplaskin.laplace.univ-tlse.fr (University of Toulouse, LAPLACE, CNRS-UPS-INP, Toulouse, France, 2008).

[22] BOLSIG+ solver ver. 03/2016, www.lxcat.net

[23] Hayashi database, www.lxcat.net, retrieved on March 14, 2017.

[24] Gibalov V.I., Pietsch G.J. // J. Phys. D: Appl. Phys. 2000. Vol. 33. P. 2618.

[25] Loeb L.B., Meek J.M. The mechanism of the electric spark. Stanford, California, USA: Stanford University Press, 1941. (Chapter II).

[26] Kettlitz M., Höft H., Holder T., Weltmann K.-D., Brandenburg R. // Plasma Sources Science and Technology. 2013. Vol. 22. P. 025003.

[27] Brandenburg R., Wagner H.-E., Morozov A.M., Kozlov K.V. // J. Phys. D: Appl. Phys. 2005. Vol. 38. P. 1649.

[28] Morgan database, www.lxcat.net, retrieved on March 14, 2017.

[29] Puech database, www.lxcat.net, retrieved on March 14, 2017.

[30] Biagi database, www.lxcat.net, retrieved on March 14, 2017.

[31] Janev R.K., Reiter D. // Phys. Plasmas. 2002. Vol. 9. P. 4071.

[32] Janev R.K., Reiter D. // Phys. Plasmas. 2004. Vol. 11. P. 780.

[33] Corrigan S.J.B. // J. Chem. Phys. 1965. Vol. 43. P. 4381.

[34] Scarduelli G., Guella G., Mancini I., Dilecce G., Benedictis S., Tosi P. // Plasma Process. Polym. 2009. Vol. 6. N 1. P. 27. 